\title{
O MONITORAMENTO COMO FERRAMENTA DA REDUÇ̃̃O DO CONSUMO DE ÁGUA POTÁVEL NA FACULDADE DE CIÊNCIAS DA ADMINISTRAÇÃO DE PERNAMBUCO - FCAP/UPE
}

\author{
Anna Elis Paz Soares ${ }^{1}$, Amanda Rafaely Monte do Prado ${ }^{1}$, Simone Rosa da Silval \\ ${ }^{1}$ Departamento de Engenharia Civil, Escola Politécnica de Pernambuco (POLI-UPE), 50720-001, Recife, Brasil. \\ *E-mail: $\underline{\text { aeps_pec@poli.br }}$
}

Recebido em: 24/02/2018 Aceito em: 01/10/2018

\section{RESUMO}

A água é um bem indispensável para o desenvolvimento econômico e humano. Porém, sua escassez vem se agravando ao longo dos anos. Diante desta problemática, as Instituições de Ensino Superior têm um papel fundamental no fomento à sustentabilidade, visando promover a reflexão e mudanças na sociedade. O presente estudo tem por objetivo diagnosticar o padrão de consumo de água e analisar o impacto dos vazamentos no consumo total da Faculdade de Ciências da Administração de Pernambuco - FCAP/UPE. A metodologia consistiu no cadastro da edificação, levantamento populacional, monitoramento do consumo de água e de patologias e cálculo dos Índices de Consumo (IC) e Vazamentos (IV). Constatou-se uma redução no indicador de consumo médio da Instituição ao longo dos anos, passando de 12 L/ag.consumidor/dia no período 2012-2014 para 9 L/ag.consumidor/dia entre 2015 e 2017. O índice médio mensal de vazamentos foi de $8 \%$, em que os ambientes utilizados pelos funcionários foram os que mais apresentaram equipamentos com patologia. Além disso, constatou-se a importância da atuação do gestor de águas na redução do consumo. Os resultados obtidos até então comprovam a importância do monitoramento do consumo de água para tomada de ações de conservação deste recurso e servirão de base para a melhoria do sistema de gestão da água na FCAP.

Palavras-chave: Sustentabilidade. Conservação de Água. Instituições de Ensino Superior.

\section{Introdução}

A água é um bem essencial à vida humana e na produção da maioria dos bens e serviços, incluindo alimentos e energia. Entretanto, se por um lado este recurso natural é indispensável para o desenvolvimento econômico e humano, o descaso em sua utilização, sem qualquer preocupação acerca de sua disponibilidade futura, é comum nas mais diversas atividades [13].

Além de fatores naturais, como os baixos índices pluviométricos, problemas na gestão das demandas hídricas também se apresentam como causadores da escassez de água potável. A gestão insustentável dos recursos hídricos pode causar graves danos à economia e à sociedade, afetando negativamente os benefícios referentes à redução da pobreza, criação de empregos e desenvolvimento [4-6].

O crescimento exagerado das demandas localizadas, como a concentração populacional em grandes centros urbanos, e a degradação da qualidade das águas devido ao aumento desordenado do processo de urbanização, industrialização e expansão agrícola também constituem fatores causadores da problemática $[2,7-8]$.

Desde 2012, a Região Nordeste do Brasil vem apresentando uma redução nos índices pluviométricos. No semiárido nordestino, os baixos índices somados às características climáticas naturais, como altas temperaturas e taxas de evapotranspiração, resultam em rios com baixa disponibilidade hídrica e intermitentes, afetando diretamente os sistemas de abastecimento [5]. O nível de armazenamento do "reservatório equivalente" da Região Nordeste - que representa a soma dos volumes dos principais reservatórios dos estados da Bahia, Ceará, Paraíba, Pernambuco e Rio Grande do Norte apresentou redução de cerca de 50\% entre os anos de 2012 e 2015 [9].

Diante desse cenário, é dever de toda a sociedade a busca por práticas sustentáveis, e das Instituições de Ensino Superior IES - o fomento a pesquisas e ações sustentáveis, visando promover a reflexão e mudança da sociedade em geral. Além de ensinar, pesquisar e disseminar ações sustentáveis, é fundamental que as IES pratiquem tais ações, sendo de sua responsabilidade a gestão eficaz da água em seus próprios espaços [10-11]. 
Nas IES, o desperdício de água é recorrente e, em se tratando de instituições públicas, os desperdícios tendem a ser ainda maiores, já que os usuários não são os responsáveis financeiros diretos pelo pagamento das contas d'água [11-12]. Por isso, é de suma importância a realização de estudos nessa tipologia predial, visando ao diagnóstico dos padrões de consumo de água e a busca por soluções mais sustentáveis.

Dada sua relevância, as práticas sustentáveis sobre a água estão ganhando destaque nas pesquisas em instituições de ensino superior. Ao analisar 42 universidades de variadas partes do mundo, um estudo [13] observou que o controle do consumo e o reuso da água são as principais medidas sustentáveis adotadas, aparecendo em torno de $22 \%$ dos casos.

No Brasil, são destaques o Programa de Uso Racional de Água da Universidade de São Paulo (PURA-USP) [10]; o PRÓ-ÁGUA, da Universidade Estadual de Campinas UNICAMP [14]; e os Programas de Uso Racional da Universidade Federal de São Carlos - UFSCar e da Universidade Federal da Bahia - UFBA [15-16], além de pesquisas focadas na redução dos desperdícios de água nos laboratórios, na viabilidade do reaproveitamento de água de bebedouros e no monitoramento e setorização do consumo em campi universitários $[8,11,17]$.

Diante do exposto, o presente estudo tem por objetivo diagnosticar o padrão de consumo de água e analisar o impacto dos vazamentos no consumo total da Faculdade de Ciências da Administração de Pernambuco - FCAP/UPE.

\section{Metodologia}

\subsection{Cadastro da edificação}

Foram realizados uma visita inicial à FCAP e o preenchimento da ficha cadastral, que levanta informações sobre mananciais utilizados no local, frequência do abastecimento e presença de hidrômetros. Também foi realizada a caracterização dos equipamentos hidrossanitários instalados no campus, quanto ao número, tipo e presença de tecnologia poupadora de água.

\subsection{Levantamento populacional}

Foi realizado através de dados obtidos nos Relatórios de Atividades da UPE, disponíveis no endereço eletrônico da Instituição.

\subsection{Monitoramento do consumo de água e de patologias}

Caracterizado por visitas semanais, às segundas e sextas-feiras, em que eram realizadas as leituras dos hidrômetros e a vistoria nos ambientes que utilizam água, visando detectar vazamentos ou mau funcionamento nos equipamentos hidrossanitários.

\subsection{Cálculo dos Índices de Consumo e de Vazamentos}

O Índice de Consumo (IC) é definido como a relação entre o volume consumido por uma população em determinado intervalo de tempo, sendo obtido em L/ agente consumidor/dia, segundo a Equação 1 [18]:

$$
\mathrm{IC}=(\mathrm{Cm} \times 1000) /(\mathrm{Na} \times \mathrm{Dm})
$$

Onde,

$\mathrm{Cm}$ - consumo mensal $\left(\mathrm{m}^{3}\right)$;

$\mathrm{Na}$ - Número de agentes consumidores;

Dm - número de dias úteis do mês.

Para obter o IC médio anual foram calculados os Índices de Consumo para cada mês e, posteriormente, foi feita uma média aritmética com os valores mensais obtidos.

Os dados do consumo mensal foram obtidos através das leituras em campo. Para os anos anteriores, houve o apoio da concessionária local, que forneceu o histórico de consumo.

O número de agentes consumidores foi obtido através dos Relatórios de Atividades da UPE, disponíveis no site da Instituição [19], para os anos de 2012 a 2016. Para 2017, considerou-se uma média dos dados dos últimos dois anos, uma vez que os números oficiais ainda não haviam sido disponibilizados. O quantitativo refere-se aos docentes, funcionários administrativos efetivos, alunos de graduação, de pós-graduação stricto e lato sensu da FCAP, e ainda aos alunos e funcionários efetivos da Escola de Aplicação do Recife. Esta é uma unidade organizacional da Faculdade de Ciências da Administração de Pernambuco, que conta com mais de 400 alunos divididos entre as turmas de ensino fundamental e ensino médio.

Para os dias úteis de cada mês foram desconsiderados domingos e feriados, e os meses de janeiro e dezembro, por apresentarem baixa atividade escolar.

O Índice de Vazamentos (IV) representa a razão entre os pontos com vazamento e os pontos de utilização total do sistema em dado mês, de acordo com a Equação 2 [20]:

$$
\mathrm{IV}=\left(\sum \mathrm{Pc}\right) /\left(\sum \mathrm{Pv}\right) \times 100
$$

Onde,

$\sum \mathrm{Pc}$ - número de pontos de utilização do sistema com vazamento no referido mês; 
$\sum \mathrm{Pv}$ - número de pontos de utilização total do sistema no referido mês.

Os dados foram obtidos através das visitas semanais realizadas durante o período de maio/2017 até novembro/2017.

\section{Resultados e discussões}

\subsection{Caracterização do local de estudo}

A Faculdade de Ciências da Administração de Pernambuco - FCAP - integra o campus Benfica da Universidade de Pernambuco - UPE, juntamente com a Escola Politécnica de Pernambuco - POLI. Sua estrutura é composta por três blocos: A, B e C - como mostra a Figura 1 - que possuem salas de aula, salas de administração, biblioteca, auditórios, copa, cantina, além de duas guaritas e área de estacionamento.

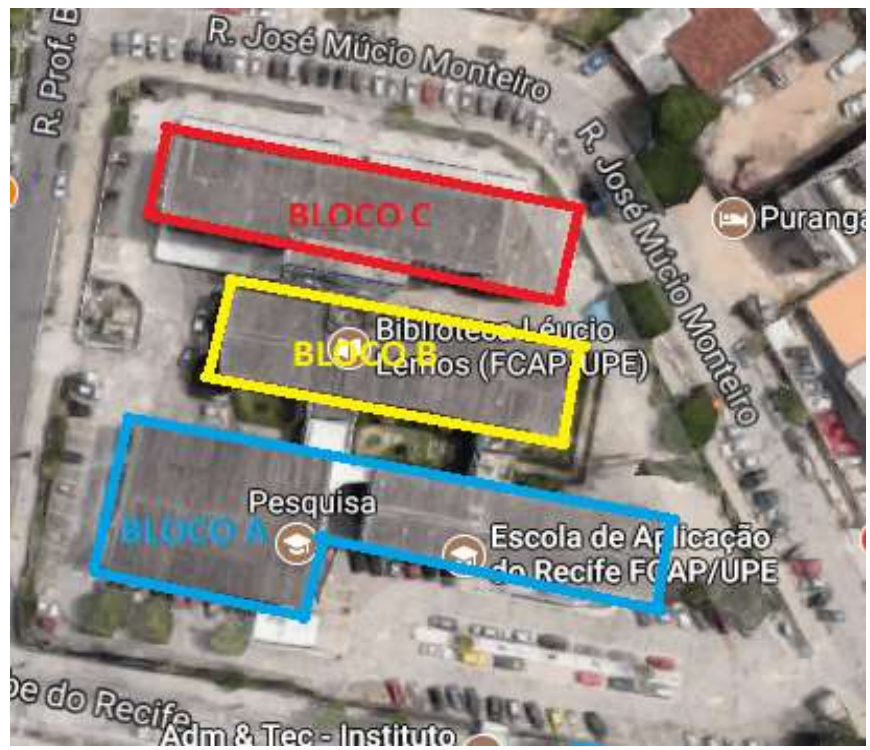

Figura 1- Vista aérea da FCAP. Fonte: Google Maps (Acesso em 07/11/2017).

O consumo de água e a população correspondente ao período entre 2012 e 2017, compreendendo funcionários efetivos e alunos de graduação, stricto sensu e lato sensu da FCAP, bem como funcionários e alunos da Escola do Recife (ER), podem ser observados na Tabela 1.

Tabela 1. População e consumo de água da FCAP entre 2012-2017.

\begin{tabular}{ccc}
\hline Ano & População & Consumo Anual $\left(\mathbf{m}^{\mathbf{3}}\right)$ \\
\hline 2012 & 2.939 & 11.423 \\
2013 & 2.742 & 10.895 \\
2014 & 2.334 & 6.511 \\
2015 & 2.099 & 5.066 \\
2016 & 1.894 & 4.857 \\
2017 & 2.007 & 4.854 \\
\hline
\end{tabular}

Através da Figura 2, pode-se observar que o número de alunos é expressivo em comparação ao número de funcionários efetivos. Os alunos de graduação correspondem a maior parcela, seguidos dos de pós-graduação e educação básica.
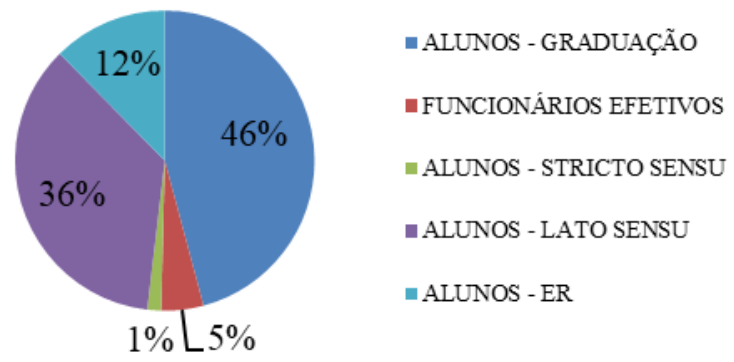

Figura 2- Distribuição da população da FCAP entre 2015-2017.

Quanto à distribuição dos equipamentos hidrossanitários, o campus possui 75 torneiras dos tipos convencional e hidromecânica; 75 vasos sanitários com caixa de descarga, válvula de descarga e caixa acoplada de simples e duplo acionamento; 15 chuveiros de materiais plástico e metálico e 10 mictórios dos tipos convencional e hidromecânico, como mostrado na Tabela 2.

Tabela 2. Quantidade de equipamentos hidrossanitários por tipologia.

\begin{tabular}{ccc}
\hline Equipamento & Tipo & Quant. \\
\hline \multirow{2}{*}{ Torneira } & Convencional & 26 \\
\cline { 2 - 3 } & Hidromecânica & 49 \\
\cline { 2 - 3 } Vaso Sanitário & 01 \\
\cline { 2 - 3 } & Caixa de descarga & 36 \\
\cline { 2 - 3 } & Cálvula de descarga & 34 \\
\cline { 2 - 3 } & Caixa acoplada acionamento simples & 04 \\
\cline { 2 - 3 } Chuveiro & Caixa acoplada acionamento duplo & 07 \\
\cline { 2 - 3 } & Plástico & 01 \\
\hline \multirow{2}{*}{ Mictório } & Metálico & 09 \\
\cline { 2 - 3 } & Convencional &
\end{tabular}

\section{2 Índice de Consumo (IC)}

A partir da análise dos dados obtidos, os ICs mensais foram calculados para cada ano, excluindo-se os meses de janeiro e dezembro, conforme descrito na metodologia.

Ao analisar a série histórica completa, foram obtidas 60 amostras, nas quais o IC máximo foi de $19 \mathrm{~L} / \mathrm{ag}$.consumidor/dia (abril/2014) e o mínimo foi de 4 L/ag.consumidor/dia (julho/2015). O IC médio para todo o período estudado foi de 11 L/ag.consumidor/dia, com um desvio padrão de \pm 3 . 


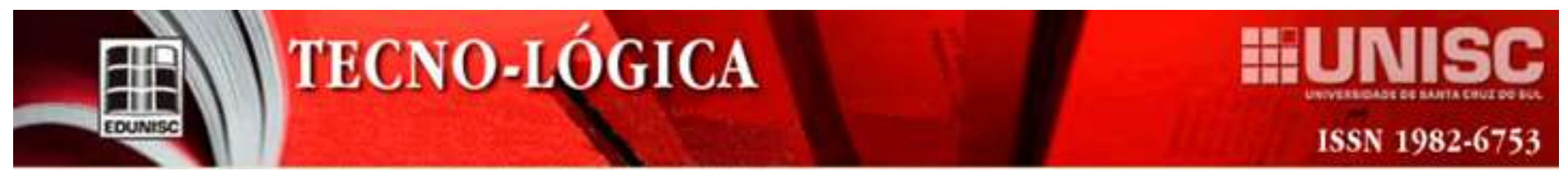

Na Figura 3 pode-se observar o comportamento do IC em cada ano do período estudado. Observa-se que, nos anos de 2012 e 2013, o IC apresentava os maiores valores médios (13 L/ag.consumidor/dia); a partir de 2014, o IC médio cai para 10 L/ag.consumidor/dia, mas ainda apresenta grande variância. Nos anos sequentes observa-se uma redução no IC médio para 9 L/ag.consumidor/dia e também uma maior homogeneidade nos valores da série, posto que, em 2017, o IC médio voltou a subir para 10 L/ag.consumidor/dia, enquanto que o ano de 2016 apresentou os melhores resultados da série.

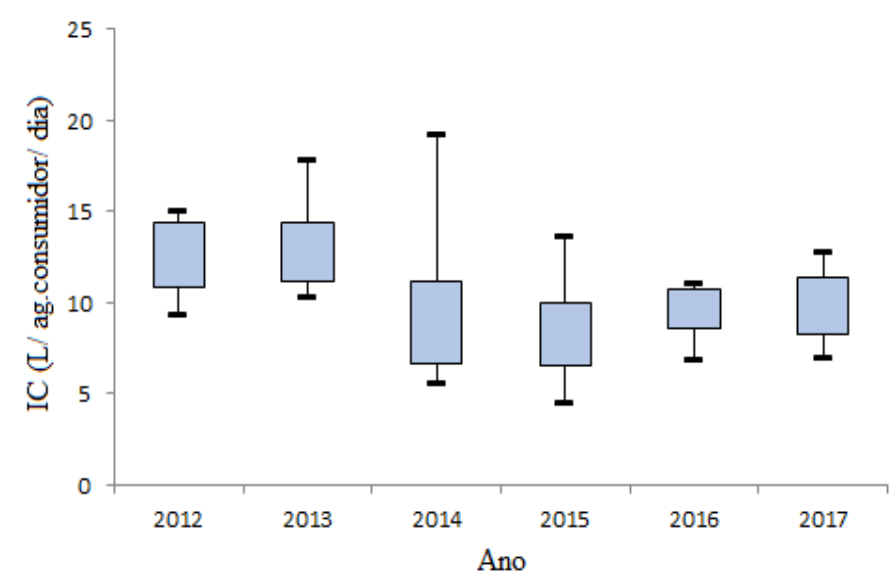

Figura 3 - Comparativo entre os indicadores de consumo de água de cada ano analisado.

A redução do IC, a partir de 2015, corresponde a uma diferença de, aproximadamente, $430 \mathrm{~m}^{3}$ de água no consumo anual. Foi observado que esse período coincidiu com o ano de instituição do cargo de gestor de águas, o que provavelmente contribuiu para a redução do consumo. Na FCAP, o gestor realiza leituras diárias do hidrômetro e as registra em planilha, mantendo-se atento às variações de consumo que alertam sobre a presença de vazamentos.

O gestor realiza inspeções periódicas nas tubulações que, em sua maioria, são antigas, e estão localizadas no subsolo, ou seja, o acesso é difícil e os vazamentos não são percebidos com facilidade.

No ano de 2017, as hipóteses para o aumento do consumo são os vazamentos que ocorreram em tubulações de difícil acesso e a um período em que estavam sendo realizadas reformas nos principais banheiros da unidade.

A Figura 4 apresenta um comparativo entre os índices do período 2012-2014 e 2015-2017, dada a importância da redução no IC ocorrida após 2014. No primeiro período, o IC médio era de 12 L/ag.consumidor/dia, no segundo período de tempo analisado, o IC foi de 9 L/ag.consumidor/dia. De um período para o outro, também é possível notar a redução da amplitude dos dados, o que demonstra uma diminuição da ocorrência de eventos extremos, geralmente causados por vazamentos de médio a grande porte ou que passaram um longo período de tempo para serem detectados ou corrigidos.

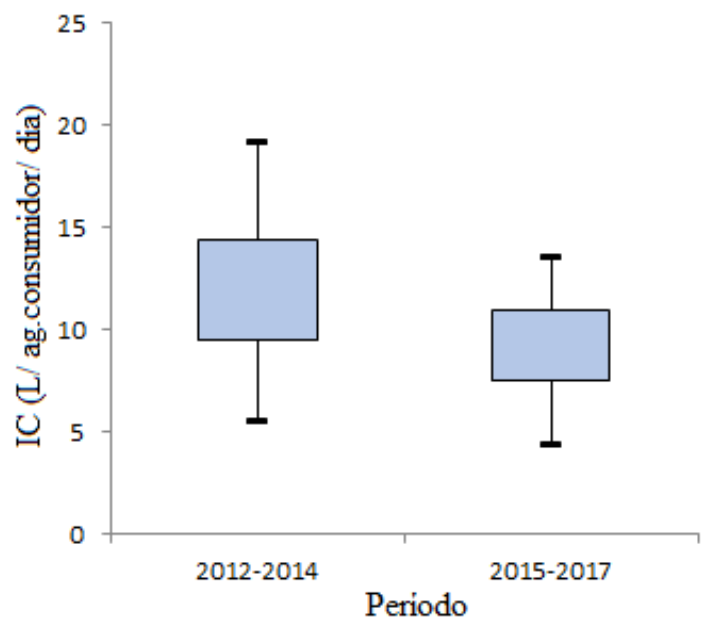

Figura 4 - Comparativo entre os indicadores de consumo por período.

Soares et al. [17] analisaram o consumo de água e determinaram os indicadores de consumo da Escola Politécnica de Pernambuco (POLI-UPE), no período 2012-2015. Juntamente com a FCAP, a POLI forma o Campus Benfica da Universidade de Pernambuco. As duas unidades estão situadas na mesma rua, mas não possuem compartilhamento da rede hidráulica ou sanitária.

Comparando os valores do IC médio anual das duas unidades, apresentados na Figura 5, observa-se que a FCAP possui índices mais elevados em todos os anos. O IC médio entre os anos de 2012-2015 da POLI é de 6 L/ ag.consumidor/dia, 57\% inferior ao valor de $11 \mathrm{~L} / \mathrm{ag}$.consumidor/dia da FCAP.

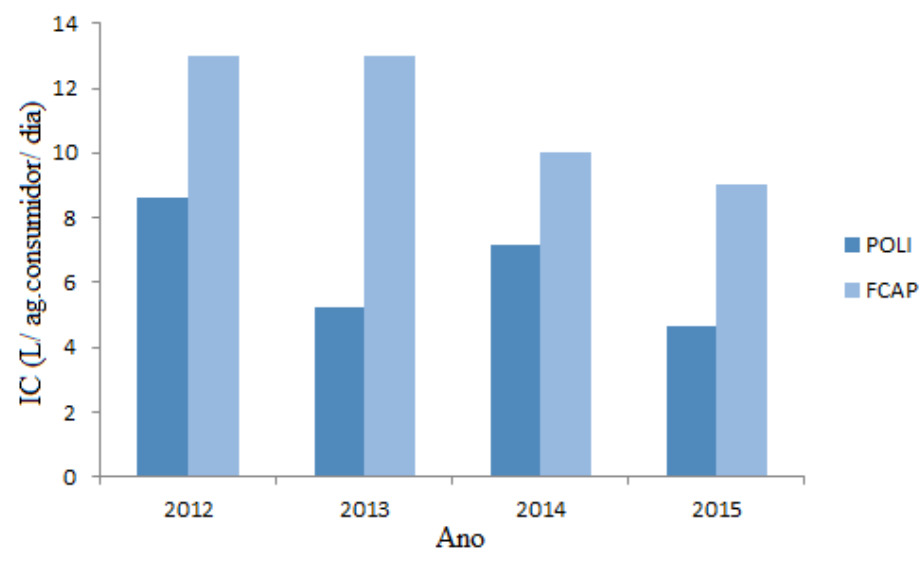

Figura 5 - Comparativo entre os Índices de Consumo da POLI e da FCAP. 
Embora as duas unidades estejam localizadas a cerca de 200 metros de distância e a população da POLI seja superior a da FCAP, existem outros fatores que influenciam no padrão de consumo de uma edificação e que precisam ser levantados e analisados para compreender a diferença nos indicadores, como por exemplo, hábitos e percepção dos usuários para o uso racional da água, índice de vazamentos e perdas, entre outros.

Na Universidade de Campina Grande, na Paraíba, o IC apontado foi de $33 \mathrm{~L} /$ pessoa/dia [21]. A Universidade de São Paulo, após a realização do Programa de Uso Racional da Água (PURA-USP) conseguiu uma redução do IC de 114 para 70 L/dia/pessoa [10]. A Universidade Federal da Bahia, por sua vez, reduziu o consumo per capita de água pela metade, de 46 para 23 L/pessoa/dia [16].

Nas Instituições de Ensino Superior em outros países, a variação do Indicador de Consumo também é expressiva, principalmente nos campi com dormitórios para o corpo discente. $\mathrm{Na}$ Universidade de Kwame Nkrumah, localizada na cidade de Kumasi, Gana, foi estimado que o uso médio de água entre os estudantes era de $115 \pm 5 \mathrm{~L} /$ pessoa/dia [22]. Na Universidade da Califórnia - Berkeley, foi apontado um IC médio de 146 L/pessoa/dia no ano de 2009 [23]. No Campus Penryn/ Tremough (Cornwall, Inglaterra, Reino Unido), o IV reportado para o ano de 2011 foi de aproximadamente 45 L/aluno/dia, considerando 200 dias letivos [24].

Mesmo sem a implantação de medidas físicas para redução do consumo de água, o Campus Benfica apresentou indicadores menores que os das universidades brasileiras e estrangeiras. No entanto, ao comparar o consumo de água do campus com outras tipologias prediais dentro do Estado de Pernambuco, os indicadores se aproximam. Nunes et al. (2018) calculou o IC de seis escolas públicas da região metropolitana de Recife, em que os valores variaram de 4 a 17 L/aluno/dia [25]. Silva (2018), ao estudar os prédios públicos administrativos de Recife, encontrou um IC médio por agente consumidor igual a 33,45 L/funcionário/dia [26]. O fato de Pernambuco ter uma das menores disponibilidades hídricas per capita do país [5] pode contribuir para que esse indicador seja menor quando comparado com outras regiões e países.

\section{3 Índice de Vazamentos (IV)}

O diagnóstico dos equipamentos hidrossanitários teve início em maio de 2017 e os resultados obtidos são apresentados abaixo (Figura 6).

Os resultados encontrados devem ser analisados em caráter preliminar, pois a série de dados possui apenas seis meses. Percebe-se uma tendência decrescente no IV a partir de junho, alcançando $6,82 \%$ em agosto. $\mathrm{O}$ mesmo comportamento pode ser observado entre os meses de setembro e outubro. O IV médio da FCAP é de $8 \%$, com apenas um mês acima da média.

Entretanto, outros fatores devem ser levados em consideração, como a quantidade de equipamentos hidrossanitários, a tipologia da Instituição e a sua população, de tal forma que é imprescindível o estudo de cada caso em particular, a fim de propor soluções específicas para cada situação.

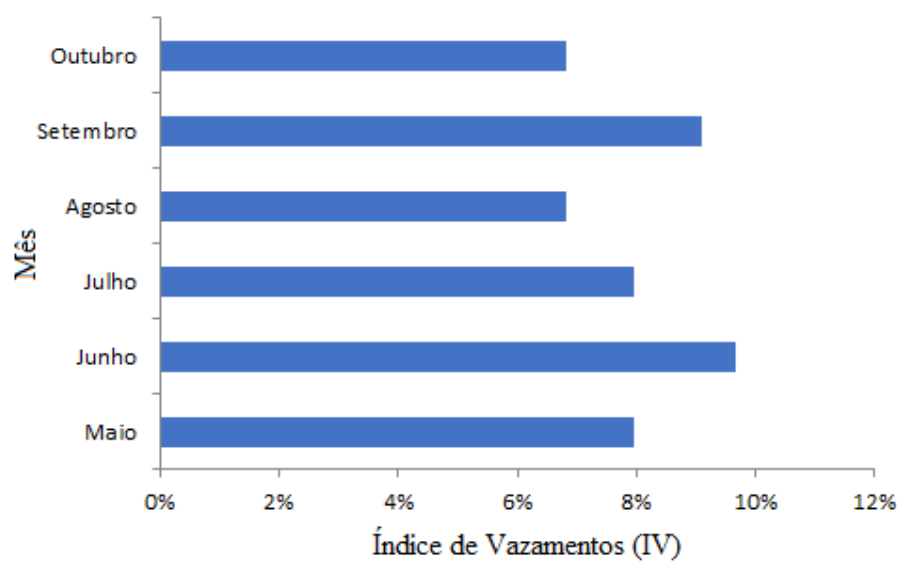

Figura 6 - IV mensal (\%).

A Figura 7 mostra a relação entre os equipamentos hidrossanitários que apresentaram vazamentos no intervalo de tempo estudado e os blocos da FCAP. Nota-se que o Bloco A, que, em geral, apresenta uso administrativo e por parte dos funcionários de limpeza e manutenção, possui a maior quantidade de patologias verificadas, enquanto que os Blocos $\mathrm{B}$ e $\mathrm{C}$, os mais utilizados pelos alunos, possuem porcentagem inferior.

\section{=BLOCOA $=B L O C O B=B L O C O C \equiv G U A R I T A S$}

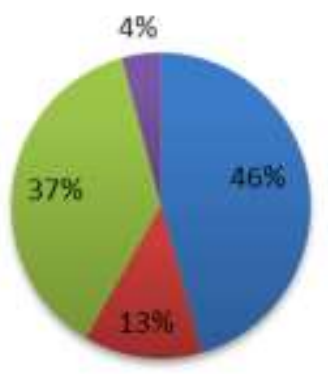

Figura 7 - Relação entre os equipamentos com vazamentos e os blocos onde estão localizados ( $\Sigma \mathrm{Pc}$ x Bloco). 
Importante ressaltar que os banheiros dos Blocos B e C passaram por reformas nos últimos anos, apresentando equipamentos com menor desgaste, o que pode corroborar com o fato de o número de patologias identificadas nesses blocos ser menor que o do Bloco A.

Nas instituições brasileiras, as patologias em sistemas hidrossanitários são apontadas como uma das principais causas do desperdício de água nos prédios. E, não raro, a substituição de equipamentos e a melhoria do setor de manutenção são apontadas como medidas para redução do consumo de água [15-18, 20-21]. Nas escolas públicas de Recife, por exemplo, foram apontados Índices de Vazamentos variando de 6 a 28\% [25]. Nos prédios públicos administrativos, o IV variou de 1 a $16 \%$, obtendo uma média de 4,5\% [26]. Já nas instituições fora do Brasil, nota-se uma maior preocupação com o comportamento do usuário perante o uso da água e não foram identificados relatos de patologias nos sistemas hidrossanitários [22-23, 27].

\section{Conclusões}

Com o desenvolvimento dessa pesquisa, foi possível perceber a importância da atuação do gestor de águas da FCAP para a diminuição dos desperdícios, uma vez que o mesmo faz o acompanhamento diário do consumo e está atento às variações para tomar providências rápidas, trabalho que contribui para a redução do consumo e, consequentemente, do IC.

Embora o Indicador de Consumo tenha decrescido no intervalo de tempo analisado, esse valor ainda pode ser reduzido. Para tanto, é aconselhável a substituição dos equipamentos hidrossanitários por aqueles com tecnologias poupadoras, bem como a realização de ações que conscientizem os usuários sobre a importância da conservação da água.

Com a ressalva de possuir caráter preliminar, pode-se concluir que os ambientes utilizados pelos funcionários são os que mais apresentam equipamentos com patologias. Além da substituição dos equipamentos, é recomendável analisar a percepção dos usuários, tanto alunos quanto professores e funcionários, para o uso racional da água e realizar campanhas educativas voltadas para cada público específico.

Os resultados obtidos até então comprovam a importância do monitoramento do consumo de água para tomada de ações de conservação deste recurso e servirão de base para a melhoria do sistema de gestão de água na FCAP.

\section{MONITORING AS A TOOL FOR THE REDUCTION OF POTABLE WATER CONSUMPTION AT FACULDADE DE CIÊNCIAS DA ADMINISTRAÇÃO DE PERNAMBUCO - FCAP/UPE}

ABSTRACT: Water is an indispensable resource for economic and human development. However, problems of scarcity have grown worse over time. Faced with this situation, institutions of higher education can play a fundamental role in fostering sustainability that can promote reflection and, ultimately, changes to society. This study seeks to diagnose the pattern of water consumption and analyze the impact of leaks on the total water consumption of the Faculdade de Ciências da Administração de Pernambuco (FCAP/UPE). The methodology consists of an analysis of the building, a population survey, monitoring of water consumption and pathologies, and calculation of Consumption Indices (CI) and Leak Indices (LI). The institution's average consumption indicator decreased over the years, from $12 \mathrm{~L} / \mathrm{user} /$ day during the period 2012-2014 to 9 L/user/day between 2015 and 2017. The average monthly leak rate was $8 \%$ and the environments used by employees were the ones that contained the most damaged equipment. In addition, the importance of the water manager's performance in reducing consumption was verified. The results obtained so far demonstrate the importance of monitoring water consumption to ensure that water conservation actions are taken and will serve as a basis for the improvement of the water management system at FCAP.

Keywords: Sustainability. Water Conservation. Institutions of higher education.

\section{Referências}

[1] TUNDISI, J. G. Novas Perspectivas para a Gestão de Recursos Hídricos. Revista USP, São Paulo, n.70, p. 24-35, 2006.

[2] World Water Assessment Programme - WWAP. The United Nations World Water Development Report 2015: Water for a Sustainable World. Paris: UNESCO, 2015.

[3] MOURA, M. R. F. de; SILVA, S. R. da; BARROS, E. X. do R. Análise de implantação de um sistema de aproveitamento de água pluvial em um empreendimento residencial na cidade de Recife-PE. Tecno-Lógica, Vol. 22, n. 1, p. $66-72,2018$

[4] BROWN, R. R.; FARRELLY, M. A. Delivering sustainable urban water management: a review of the hurdles we face. Water Science \& Technology WST, Vol. 59, n. 5, p. 839-846, 2009.

[5] AGÊNCIA NACIONAL DE ÁGUAS (ANA). Conjuntura dos Recursos Hídricos no Brasil. Encarte sobre a Crise Hídrica. Brasília, 2014. 
[6] World Water Assessment Programme - WWAP. Relatório Mundial das Nações Unidas sobre Desenvolvimento dos Recursos Hídricos 2016: Água e emprego. Resumo executivo. Itália: UNESCO, 2016.

[7] GUEDES, M. J. F.; RIBEIRO, M. M. R.; VIEIRA, Z. M. de C. L. Alternativas de gerenciamento da demanda de água na escala de uma cidade. Revista Brasileira de Recursos Hídricos, Vol. 19, n. 3, p. 51-62, 2014.

[8] FERREIRA, I. F.; ARAÚJO, L. A.; FRANÇA, P. H. B.; ALMEIDA, T. N.; COSTA, V. H. C.; LIMA, S. F. Análise da Viabilidade do Reaproveitamento da Água dos Bebedouros no Centro Universitário Tiradentes. Ciências Exatas e Tecnológicas, Maceió, Vol.3, n.2, p. 29-40, 2016.

[9] AGÊNCIA NACIONAL DE ÁGUAS (ANA). Conjuntura dos Recursos Hídricos no Brasil. Brasília, 2016.

[10] SILVA, G.S.; TAMAKI, H. O.; GONÇALVES, O. M. O PURA-USP e o Uso Sustentável da Água na Universidade de São Paulo. In: ENCONTRO LATINO AMERICANO DE UNIVERSIDADES SUSTENTÁVEIS - ELAUS, 1., 2008, Passo Fundo, Anais... Passo Fundo: UPS, 2008.

[11] CARLI, L. N.; DE CONTO, S. M.; BEAL, L. L.; PESSIN, N. Racionalização do Uso da Água em uma Instituição de Ensino Superior - Estudo de Caso da Universidade de Caxias do Sul. Revista de Gestão Ambiental e Sustentabilidade, São Paulo, Vol. 2, n. 1, p. 143-165, 2013

[12] YWASHIMA, L.A.; ILHA, M. S. O.; CRAVEIRO, S. G.; GONÇALVES, O. M. Método para Avaliação da Percepção dos Usuários para o Uso Racional das Águas em Escolas. In: ENCONTRO NACIONAL DE TECNOLOGIA NO AMBIENTE CONSTRUÍDO, 11., 2006, Florianópolis. Anais... Florianópolis: ANTAC, 2006.

[13] TAUCHEN, J.; BRANDLI, L. L. A Gestão Ambiental em Instituições de Ensino Superior: Modelo para Implantação em Campus Universitário. Gestão \& Produção, São Paulo, Vol. 13, n. 3, p. 503-515, 2006

[14] NUNES, S. da S. Estudo da conservação de água em edifícios localizados no Campus da Universidade Estadual de Campinas. Dissertação de Mestrado em Engenharia Civil. Universidade Estadual de Campinas. Campinas, SP. 2000

[15] AMORIM, S.V.; DIAS, L. F. S.; PAOLI, F. Uso racional da água no campus da Universidade Federal de São Carlos. In: IX ENCONTRO NACIONAL DE TECNOLOGIA DO AMBIENTE CONSTRUíDO, 09., 2002 Foz do Iguaçu. Anais... Foz do Iguaçu: ANTAC, 2002.

[16] MARINHO, M.; GONÇALVES, M. do S.; KIPERSTOK, A. Water conservation as a tool to support sustainable practices in a Brazilian public university. Journal of Cleaner Production, Vol. 62, p. 98-106, 2014.

[17] SOARES, A. E. P.; SILVA, T. L.; SILVA, S. R.; NUNES, L. G. C. F.; SILVA, J. K. Caracterização do Consumo de Água em uma Universidade Pública do Recife. In: Congresso Brasileiro de Engenharia Sanitária e Ambiental, 29., 2017, São Paulo. Anais.... São Paulo: ABES, 2017.

[18] OLIVEIRA, L. H.; GONÇALVES, O. M. Metodologia para a implantação de programa de uso racional de água em edifícios. Boletim Técnico da USP. Universidade de São Paulo. São Paulo, 1999.

[19] Site oficial da Universidade de Pernambuco. Relatório de Atividades. Disponível em: <http://www.upe.br/relatorio-de-atividades>. Acesso em $10 / 09 / 2017$

[20] GONÇALVES, O.; ILHA, M.; AMORIM, S.; PEDROSO, L. Indicadores de uso racional de água para escolas de ensino fundamental e médio. Ambiente Construído, Vol. 5, n. 3, p. 35-48, 2005.

[21] GOMES, V. L. Uso eficiente de água em campus universitário: o caso da Universidade Federal De Campina Grande. 2013. 114 p. Dissertação (Mestrado em Engenharia Civil e Ambiental) - Universidade Federal de Campina Grande, Campina Grande, 2013.
[22] ODURO-KWARTENG, S. et al. Water conservation potential in educational institutions in developing countries: case study of a university campus in Ghana. Urban Water Journal, London, Vol. 6, n. 6, p. 449-455, 2009.

[23] University of California, Berkeley. UC Berkeley water usage \& conservation study report 2010. Disponível em: < https://sustainability.berkeley.edu/>. Acesso em: 20/10/2017.

[24] Falmouth University. A Waste and Resources Management Strategy for the Tremough Campus - Version 1. Disponível em: 〈http://www.fxplus.ac.uk>. Acesso em 17/09/2017.

[25] NUNES, L.G. C. F.; SOARES, A. E. P. S.; SILVA, J. K.; SILVA, S. R. Rational water use indicators for public schools in Recife, Brazil. Water Science \& Technology: Water Supply, Vol. 18, 2018.

[26] SILVA, A. F. A. da. Análise de viabilidade técnica e econômica de medidas de conservação da água em prédios públicos administrativos do Estado de Pernambuco. 2018. 148f. Dissertação (Mestrado em Engenharia Civil) - Escola Politécnica da Universidade de Pernambuco, Recife, 2018.

[27] BONNET, J. F. et al. Analysis of electricity and water end-uses in university campuses: case-study of the University of Bordeaux in the framework of the Ecocampus European Collaboration. Journal of Cleaner Production, Vol. 10, n. 1, p. 13-24, 2002. 